\title{
Surveillance of HIV and syphilis infections among antenatal clinic attendees in Tanzania-2003/2004
}

\author{
Roland O Swai ${ }^{1}$, Geofrey R Somi G1 ${ }^{1}$ Mecky IN Matee*2, Japhet Killewo², Eligius F Lyamuya ${ }^{2}$, \\ Gideon Kwesigabo ${ }^{2}$, Tuhuma Tulli ${ }^{1}$, Titus K Kabalimu ${ }^{3}$, Lucy Ng'ang'a ${ }^{4}$, Raphael Isingo ${ }^{5}$ and \\ Joel Ndayongeje ${ }^{1}$
}

Address: ${ }^{1}$ National AIDS Control Programme, Dar es Salaam, Tanzania, ${ }^{2}$ Muhimbili University College of Health Sciences, Dar es Salaam, Tanzania, ${ }^{3}$ Commission for Science and Technology, Dar es Salaam, Tanzania, ${ }^{4}$ Centres for Disease Control- Tanzania AIDS, Dar es Salaam, Programme, Tanzania and ${ }^{5}$ National Institute for Medical Research, Dar es Salaam, Tanzania

Email: Roland O Swai - swairo@nacptz.org; Geofrey R Somi G - g_somi@yahoo.co.uk; Mecky IN Matee* - mmatee@muchs.ac.tz; Japhet Killewo - jkillewo@muchs.ac.tz; Eligius F Lyamuya - elyamuya@muchs.ac.tz; Gideon Kwesigabo - Gkwesigabo@muchs.ac.tz; Tuhuma Tulli - tuhumat@yahoo.com; Titus K Kabalimu - tkabalimu@costech.or.tz; Lucy Ng'ang'a - ngangal@tz.cdc.gov; Raphael Isingo - rrisingo@yahoo.co.uk; Joel Ndayongeje - ndayongeje@nacptz.org

* Corresponding author

Published: 10 April 2006

Received: 23 August 2005

BMC Public Health 2006, 6:9| doi:|0.1|86/|47|-2458-6-9|

Accepted: 10 April 2006

This article is available from: http://www.biomedcentral.com/I47I-2458/6/9I

(c) 2006 Swai et al; licensee BioMed Central Ltd.

This is an Open Access article distributed under the terms of the Creative Commons Attribution License (http://creativecommons.org/licenses/by/2.0), which permits unrestricted use, distribution, and reproduction in any medium, provided the original work is properly cited.

\begin{abstract}
Background: This paper presents the prevalence of human immunodeficiency virus (HIV) and syphilis infections among women attending antenatal clinics (ANC) in Tanzania obtained during the 2003/2004 ANC surveillance.

Methods: Ten geographical regions; six of them were involved in a previous survey, while the remaining four were freshly selected on the basis of having the largest population among the remaining 20 regions. For each region, six ANC were selected, two from each of three strata (urban, peri-urban and rural). Three of the sites did not participate, resulting into 57 surveyed clinics. 17,813 women who were attending the chosen clinics for the first time for any pregnancy between October 2003 and January 2004. Patient particulars were obtained by interview and blood specimens were drawn for HIV and syphilis testing. HIV testing was done anonymously and the results were unlinked.

Results: Of the 17,8I3 women screened for HIV, I,545 (8.7\% (95\% Cl = 8.3-9.I)) tested positive with the highest prevalence in women aged $25-34$ years (1 I\%), being higher among single women $(9.7 \%)$ than married women $(8.6 \%)(p<0.07)$, and increased with level of education from $5.2 \%$ among women with no education to $9.3 \%$ among those at least primary education $(\mathrm{p}<0.00 \mathrm{I})$. Prevalence ranged from $4.8 \%(95 \% \mathrm{Cl}=3.8 \%-9.8 \%)$ in Kagera to $15.3 \%(95 \% \mathrm{Cl}=13.9 \%-16.8 \%)$ in Mbeya and was; $3.7 \%, 4.7 \%, 9.1 \%, 11.2 \%$ and $15.3 \%$ for rural, semiurban, road side, urban and $15.3 \%$ border clinics, respectively $(\mathrm{p}<0.00 \mathrm{I})$.

Of the 17,323 women screened for syphilis, $1265(7.3 \%(95 \% \mathrm{Cl}=6.9-7.7))$ were positive, with highest prevalence in the age group $35-49$ yrs $(10.4 \%)(p<0.00 I)$, and being higher among women with no education than those with some education $(9.8 \%$ versus $6.8 \%)(\mathrm{p}<0.000 \mathrm{I})$, but marital status had no influence. Prevalence ranged from $2.1 \%(95 \% \mathrm{Cl}=1.4 \%-3.0 \%)$ in Kigoma to $14.9 \%(95 \% \mathrm{Cl}=13.3 \%-16.6 \%)$ in Kagera and was $16.0 \%(95 \% \mathrm{Cl}=$ $13.3-18.9), 10.5 \%(95 \% \mathrm{Cl}=9.5-1 \mathrm{I} .5)$ and $5.8 \%(95 \% \mathrm{Cl}=5.4-6.3)$ for roadside, rural and urban clinics, respectively. Syphilis and HIV co-infection was seen in I30/178I3 (0.7\%).

Conclusion: The high HIV prevalence observed among the ANC clinic attendees in Tanzania call for expansion of current voluntary counselling and testing (VCT) services and access to antiretroviral drugs (ARV) in the clinics. There is also a need for modification of obstetric practices and infant feeding options in HIV infection in order to prevent mother to child transmission of HIV. To increase uptake to HIV testing the opt-out strategy in which all clients are offered HIV testing is recommended in order to meet the needs of as many pregnant women as possible.
\end{abstract}




\section{Background}

The first AIDS case in Tanzania was reported in the Kagera region in 1983 [1]. In the late 1980's sentinel surveillance activities in antenatal clinics (ANC) were initiated in the Kagera region. Subsequently, in the early 1990s, basing on the assumption that pregnant women attending ANC are representative of the general adult population of both males and females in the reproductive age groups, the NACP developed a protocol for ANC HIV and syphilis sero-surveillance, and expanded activities to 11 of the then 20 regions of mainland Tanzania. This protocol was implemented until 1999, when the NACP undertook a comprehensive review that resulted in improved methods for HIV and syphilis surveillance.

In 1999, the NACP revised the protocol for ANC surveillance and came up with new methods of conducting sentinel surveillance that included the introduction of a 3month data collection period to replace the previous system of continuous data collection, the introduction of a technology of using dried blood spots (DBS) filter paper cards for blood storage and the standardization of HIV test approaches and quality assurance system.

The first ANC surveillance that used this strategy was conducted in 2000/2001.

The second survey using the new approach was conducted between October 2003 and January 2004 at 57 ANC sites located in ten regions of Tanzania. Similar to the previous sero-surveillance round [2], clinics in selected regions were stratified into three strata of urban, peri-urban and rural to allow the output to be presented according to these strata.

The paper presents the prevalence of human immunodeficiency virus (HIV) and syphilis infections among women attending antenatal clinics (ANC) in Tanzania obtained during the 2003/2004 ANC surveillance.

\section{Methods}

A sero-survey was conducted between October 27, 2003 and January 23, 2004 among pregnant women attending selected ANC clinics in Tanzania. This was the second of a series of sentinel sero-surveys involving pregnant women that was started in 2001/2002. The methods used in the two surveys were similar.

\section{Site selection and study population}

In 1996 Tanzania Demographic and Health Surveys (DHS) clustered regions of Tanzania mainland into six geographical zones [3]. The six zones were Coastal, Northern Highland, Lake, Central, Southern Highland and Southern. Site selection for the 2001/2 surveillance round was based on the location of the six zones from each of which one region was selected on the basis of prior participation in national ANC sentinel surveillance studies and availability of relevant data on HIV prevalence. For the current survey-round (2003/4), ten regions were selected, six of them being those surveyed in 2001/2002 while the remaining four were freshly selected on the basis of having the largest population among the remaining regions. Thus, regions with largest populations were preferentially selected.

Subsequent to the selection of the ten regions, six ANC clinic sites were selected from each region on the basis of a large catchment population, high average number of monthly bookings, road accessibility and previous participation in national sentinel surveillance studies. Hence, a total of sixty sites were obtained. However, three of the sites did not participate in the training exercise for the surveillance protocol and were therefore excluded from the study. The 57 sites included in the study were stratified into three distinct geographical categories for each region according to whether they were urban, peri-urban or rural with the following definition:

- Urban - ANC clinic is located within the regional headquarter town

- Peri-urban - ANC clinic is located in a town other than the regional headquarter town

- Rural - ANC clinic is located in a rural area.

Peri-urban sites located along major roads that cross either a border town or the region were classified as border sites or roadside sites, respectively. The study population consisted of all the pregnant women presenting for the first time to the selected ANC clinics for any pregnancy during the surveillance period of three months.

\section{Specimen collection and transportation}

After obtaining an informed verbal consent from the pregnant woman at the clinic, about 3-5 ml of blood was collected aseptically by venepuncture in EDTA vacutainer tubes for RPR testing. The syphilis result was recorded on the woman's clinic card and on the surveillance data collection form. The remaining blood specimen was then used to prepare DBS for HIV testing whereby consent was not needed. ANC staff applied $100 \mu \mathrm{l}$ of whole blood to each of five circles on a DBS card (labeled with a unique surveillance number), which was then left to dry at room temperature. At this point the upper part of the surveillance data form that contains a woman's clinic card number is removed and destroyed. ANC staff mailed completed data collection forms and DBS for HIV testing using weekly courier service to the National HIV Reference Laboratory (NHRL) at MUCHS. 


\section{Syphilis testing and treatment}

In all study sites rapid plasma reagin (RPR) test was done on the site. In the majority of rural sites, ANC nurse performed the test whereas, in most of the urban and periurban sites it was carried out in a laboratory by a Laboratory Technician/Assistant. Results were recorded directly on the data collection form and on woman's clinic card or laboratory investigation request form. No TPP or TPHA was done on the positives RPR samples for confirmation of test results or quality assurance. Women whose RPR test results were positive were offered treatment based on the National STD Treatment Guidelines [4].

\section{HIV testing}

At the National HIV Reference laboratory, dried blood was eluted from the DBS card and tested using Vironostika ${ }^{\circledR}$ HV Uni-Form II Ag/Ab ELISA test (Biomerieux, The Netherlands). Specimens with negative results underwent no further testing and were considered negative. Specimens positive on the first ELISA were retested using a second ELISA test, Vironostika ${ }^{\circledR}$ HIV Uni-Form II Plus O (Biomerieux, The Netherlands) on the same DBS card. The ELISA algorithm was independently validated by CDC in Atlanta and by the NHRL at MUCHS. Specimens that reacted positive on the second test were considered positive. Specimens that reacted negative on the second test were considered negative.

\section{Quality assurance}

Ten per cent of all samples were randomly selected by MUCHS for quality assurance (QA) testing at a laboratory different from the one which carried out the initial testing. Every 10th sample starting from number 01 at each site was selected.

\section{Ethical considerations}

The Ministry of Health awarded ethical clearance to the National HIV Surveillance protocol. Because HIV test results were not linked by name and tests were performed on residual blood from routine syphilis screening, obtaining informed consent was not warranted. All information linking the sample to the client was removed and DBS HIV testing occurred anonymously. Women who wished to know their HIV status were referred to a nearby voluntary counseling and testing (VCT) facility.

\section{Data entry and analysis}

Laboratory technologists entered HIV test results in laboratory log books and delivered them to NACP. NACP data entry clerks performed double entry of data manually into Epi-Info Programme [5]. The two files were validated and incorrect entries were corrected.

Data were analysed by a team of researchers from NACP, MUCHS, NIMR and CDC, initially during a weeklong analysis meeting, followed by several rounds of consensus gathering and review. ANC HIV and syphilis prevalence rates were calculated by age, marital status, parity, educational level, and distance from residence to ANC and duration in residence. Prevalence were calculated with 95\% confidence intervals (CI) to guide interpretation. Data were analysed using the statistical software packages EpiInfo 2002 [5] and Stata for windows Version 8.0 [6].

\section{Results \\ HIV prevalence}

A total of 17,813 antenatal clinic attendees were enrolled in the ANC serosurveillance study from 57 clinics located in 10 regions of Tanzania between October 27th 2003 and January $23^{\text {rd }} 2004$. The number of enrolled women regionally ranged from 1135 in Lindi to 3018 in Dar es Salaam. A total of 1,545 women tested HIV positive resulting in an overall HIV prevalence in this population of $8.7 \%(95 \% \mathrm{CI}=8.3,9.1)$. HIV infection prevalence ranged from a low of $4.7 \%(95 \% \mathrm{CI}=3.8,9.8 \%)$ in Kagera region to a high of $15.7 \%(95 \% \mathrm{CI}=13.9-16.8)$ in Mbeya region (Fig 1). HIV prevalence is also presented at the clinic level (Table 1).

Of the 57 ANC sites surveyed in this study, $13(22.8 \%)$ were found to have a prevalence of HIV infection of $10 \%$ or more. These high prevalence sites consisted of one clinic in Dodoma (urban), three urban clinics in Dar es Salaam, two clinics in Lindi (urban), one in Morogoro (urban), one in Tanga (urban) and five in Mbeya (2 urban, 1 rural, 1 border and 1 road side) (Table 1 ).

The HIV prevalence differed according to residence ranging between $3.7 \%$ for rural clinics, $4.7 \%$ for semi-urban, $9.1 \%$ for road side, $11.2 \%$ for urban and $15.3 \%$ for border clinics ( $<<0.001)$ (Figure 2).

In all regions, HIV prevalence was highest among women aged 25 - 34 years. Rates were similar among the youngest and oldest age groups (Fig 3). HIV prevalence among single women $(9.7 \%)$ was slightly higher but not statistically different from that of married women $(8.6 \%)(\mathrm{p}<0.07)$, (Fig 4).

HIV prevalence increased with level of education from $5.2 \%$ among women with no education to $9.3 \%$ among those with some primary education or more $(\mathrm{p}<0.001)$ (Fig 5).

\section{Syphilis prevalence}

A total of 17,323 ANC attendees were tested for syphilis during the study period. A total of 1,265 women tested positive, an overall syphilis prevalence of $7.3 \%$ (95\% CI = 6.9-7.7) (Fig 1). Syphilis infection prevalence ranged from a low of $2.1 \%(95 \% \mathrm{CI}=1.4,3.0)$ in Kigoma region 


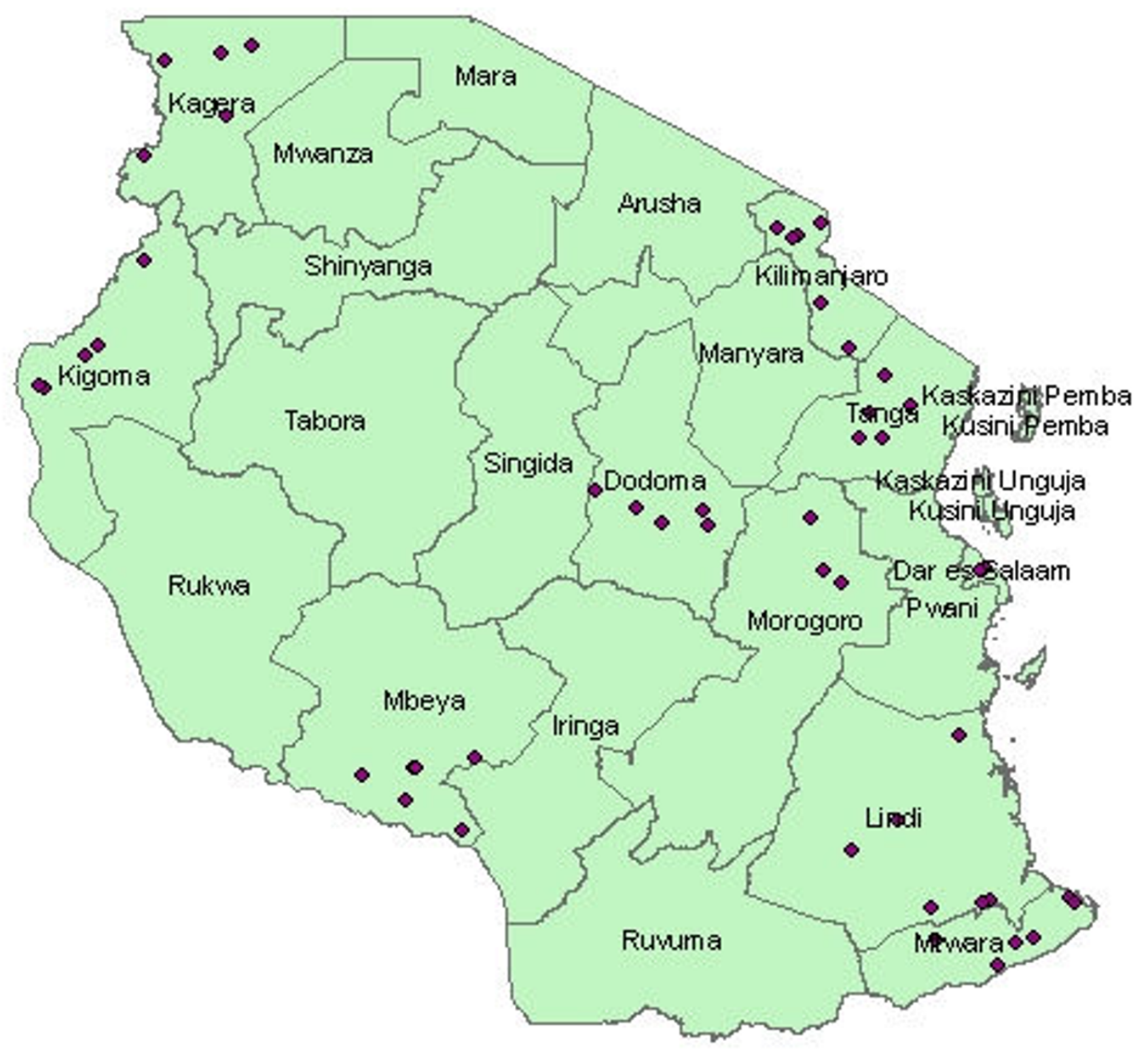

Figure I

Map of Tanzania study showing study sites.

to a high of $14.9 \%(95 \% \mathrm{CI}=13.3,16.6)$ in Kagera region where interestingly, the HIV prevalence was found to be the lowest (Fig 1).

The prevalence of syphilis was highest among attendees from roadside clinics $16.0 \%(95 \% \mathrm{CI}=13.3-18.9)$ than those from rural clinics $10.5 \%(95 \% \mathrm{CI}=9.5-11.5)$ and lowest among urban clinic attendees 5.8\% (95\% CI = 5.46.3) (Fig 2). Women living in rural areas had higher prev- alence than those in urban areas $(\mathrm{p}<0.0001)$ (Figure 6). Marital status did not appear to influence the prevalence of syphilis (Fig 4). The age specific prevalence of syphilis were $6.5 \%$ for age group $15-24,7.8 \%$ for age group $25-$ 34 and $10.4 \%$ for age group 35-49 (Fig 3). The observed differences in age-specific prevalence were statistically significant ( $\mathrm{p}<0.001)$, suggesting that there was a higher likelihood of having syphilis for women aged $>34$ years compared to those less than 34 years of age (Fig 3 ). As in 
Table I: Prevalence of HIV and syphilis infection by ANC sites, Tanzania 2003/04

\begin{tabular}{|c|c|c|c|c|c|c|c|c|}
\hline \multirow[b]{2}{*}{ Site } & \multicolumn{4}{|l|}{ RPR } & \multicolumn{4}{|l|}{ HIV } \\
\hline & Total & Positive & \% Prevalence & $95 \% \mathrm{Cl}$ & Total & Positive & $\%$ prevalence & $95 \% \mathrm{Cl}$ \\
\hline Dar es salaam & 2959 & $|5|$ & 5.1 & $4.3-6.0$ & 3018 & 325 & 10.8 & 9.7 - I I.9 \\
\hline cBuguruni & 879 & 11 & 1.3 & $0.6-2.2$ & 884 & 107 & 12.1 & $10.0-14.4$ \\
\hline cKasorobo & 446 & 46 & 10.3 & $7.7-13.5$ & 450 & 43 & 9.6 & $7.0-12.7$ \\
\hline cKigamboni & 386 & 4 & 1.0 & $0.3-2.6$ & 389 & 36 & 9.3 & $6.6-12.6$ \\
\hline cOysterbay & 466 & 37 & 7.9 & $5.7-10.8$ & 478 & 50 & 10.5 & $7.9-13.6$ \\
\hline cKimara & 361 & 10 & 2.8 & $1.3-5.0$ & 384 & 35 & 9.1 & $6.4-12.4$ \\
\hline ‘Kiwalani & 421 & 43 & 10.2 & $7.5-13.5$ & 433 & 54 & 12.5 & $9.5-16.0$ \\
\hline Dodoma & 1597 & 134 & 8.4 & $7.1-9.9$ & $|62|$ & 126 & 7.8 & $6.5-9.2$ \\
\hline aBahi & 176 & 6 & 3.4 & $1.3-7.3$ & 181 & 8 & 4.4 & $1.9-8.5$ \\
\hline aHandali & 228 & 64 & 28.1 & $22.3-34.4$ & 228 & 1 & 0.4 & $0.0-2.4$ \\
\hline eKibaigwa & 203 & 5 & 2.5 & $0.8-5.7$ & 203 & 18 & 8.9 & $5.3-13.7$ \\
\hline cMakole & 331 & 4 & 1.2 & $0.3-3.1$ & 339 & 31 & 9.1 & $6.3-12.7$ \\
\hline bMpwapwa & 308 & 29 & 9.4 & $6.4-13.2$ & 319 & 19 & 5.9 & $3.6-9.1$ \\
\hline cWajenzi & 351 & 26 & 7.4 & $4.9-10.7$ & 351 & 49 & 13.9 & $10.5-18.0$ \\
\hline Kagera & 1814 & 270 & 14.9 & $13.3-16.6$ & 1826 & 87 & 4.7 & $3.8-5.8$ \\
\hline cBukoba & 409 & 21 & 5.1 & $3.2-7.7$ & 410 & 39 & 9.5 & $6.9-12.8$ \\
\hline bKatoro & 244 & 64 & 26.2 & $20.8-32.2$ & 245 & 6 & 2.4 & $0.9-5.3$ \\
\hline aKimeya & 235 & 51 & 21.7 & $16.6-27.5$ & 241 & 2 & 0.8 & $0.1-3.0$ \\
\hline aNkwenda & 451 & 86 & 19.1 & $15.5-23.0$ & 452 & 7 & 1.5 & $0.6-3.2$ \\
\hline bNyamiaga & 180 & 27 & 15.0 & $10.1-21.1$ & 182 & 4 & 2.2 & $0.6-5.5$ \\
\hline cRwamishenye & 295 & 21 & 7.1 & $4.5-10.7$ & 296 & 29 & 9.8 & $6.7-13.8$ \\
\hline Kilimanjaro & 1405 & 89 & 6.3 & $5.1-7.7$ & 1429 & 81 & 5.7 & $4.5-7.0$ \\
\hline eHedaru & 219 & 84 & 38.4 & $31.9-45.1$ & 221 & 5 & 2.3 & $0.7-5.2$ \\
\hline cMajengo & 323 & 1 & 0.3 & $0.0-1.7$ & 323 & 22 & 6.8 & $4.3-10.1$ \\
\hline aMasama & 266 & 0 & 0 & $0.0-1.4$ & 268 & 17 & 6.3 & $3.7-10.0$ \\
\hline aUmbwe & 103 & 0 & 0 & $0.0-3.5$ & 103 & 3 & 2.9 & $0.6-8.3$ \\
\hline bHuruma & 187 & 2 & 1.1 & $0.1-3.8$ & 323 & 26 & 8.1 & $5.3-11.6$ \\
\hline cPasua & 307 & 2 & 0.7 & $0.1-2.3$ & 191 & 8 & 4.2 & $1.8-8.1$ \\
\hline Mbeya & 2386 & 149 & 6.2 & $5.3-7.3$ & 2442 & 384 & 15.7 & $14.3-17.2$ \\
\hline eChimala & 249 & 18 & 7.2 & $4.3-11.2$ & 249 & 38 & 15.3 & $11.0-20.3$ \\
\hline allembo & 178 & 13 & 7.3 & $3.9-12.2$ & 188 & 15 & 8.0 & $4.5-12.8$ \\
\hline cKiwanjampaka & 724 & 29 & 4.0 & $2.7-5.7$ & 726 & 137 & 18.9 & $16.1-21.9$ \\
\hline dKyela & 315 & 22 & 7.0 & $4.4-10.4$ & 323 & 51 & 15.8 & $12.0-20.2$ \\
\hline algamba & 195 & 29 & 14.9 & $10.2-20.7$ & 228 & 24 & 10.5 & $6.9-15.3$ \\
\hline cRuanda & 725 & 38 & 5.2 & $3.7-7.1$ & 728 & 116 & 15.9 & $13.3-18.8$ \\
\hline Mtwara & 1262 & 115 & 9.1 & $7.6-10.8$ & 1279 & 66 & 5.1 & $4.0-6.5$ \\
\hline cLigula & 388 & 29 & 7.5 & $5.1-10.6$ & 389 & 37 & 9.5 & $6.8-12.9$ \\
\hline bMangaka & 264 & 21 & 8.0 & $5.0-11.9$ & 267 & 13 & 4.9 & $2.6-8.2$ \\
\hline aNanyamba & 173 & 9 & 5.2 & $2.4-9.6$ & 176 & 6 & 3.4 & $1.3-7.3$ \\
\hline bTandahimba & 146 & 8 & 5.5 & $2.4-10.5$ & 147 & I & 0.7 & $0.0-3.7$ \\
\hline cLikombe & 165 & 29 & 17.6 & $12.1-24.3$ & 165 & 7 & 4.2 & I.7- 8.5 \\
\hline aMkunya & 126 & 19 & 15.1 & $9.3-22.5$ & 135 & 2 & 1.5 & $0.2-5.2$ \\
\hline Kigoma & 1246 & 26 & 2.1 & $1.4-3.0$ & 1349 & 69 & 5.1 & $4.0-6.4$ \\
\hline bKibondo & 204 & 0 & 0.0 & $0.0-1.8$ & 217 & 7 & 3.2 & $1.3-6.5$ \\
\hline bKiganamo & 293 & 5 & 1.7 & $0.6-3.9$ & 326 & 11 & 3.4 & $1.7-6.0$ \\
\hline cKigoma & 296 & 6 & 2.0 & $0.7-4.4$ & 301 & 21 & 7.0 & $4.4-10.5$ \\
\hline aNyakitonto & 113 & 2 & 1.8 & $0.2-6.2$ & 163 & 6 & 3.7 & $1.4-7.8$ \\
\hline${ }^{c} U_{j i j i}$ & 340 & 13 & 2.8 & $2.1-6.4$ & 342 & 24 & 7.0 & $4.5-10.3$ \\
\hline Lindi & $1 / 12$ & 79 & 7.1 & $5.7-8.8$ & $1 / 35$ & 80 & 7.1 & $5.6-8.7$ \\
\hline aChumo & 132 & 4 & 3.0 & $0.8-7.6$ & $|4|$ & 4 & 2.8 & $0.8-7.1$ \\
\hline bLiwale & 296 & 7 & 2.4 & $1.0-4.8$ & 307 & 8 & 2.7 & $||-.5 . \mid$ \\
\hline
\end{tabular}


Table I: Prevalence of HIV and syphilis infection by ANC sites, Tanzania 2003/04 (Continued)

\begin{tabular}{|c|c|c|c|c|c|c|c|c|}
\hline bNachingwea & 227 & 32 & 14.1 & $9.8-19.3$ & 227 & 22 & 9.7 & $6.2-14.3$ \\
\hline aNyangao & 231 & 20 & 8.7 & $5.4-13.1$ & 233 & 12 & 5.2 & $2.7-8.8$ \\
\hline cSokoine & 117 & 7 & 6.0 & $2.4-11.9$ & 119 & 16 & 13.5 & $7.9-20.9$ \\
\hline cTown clinic & 109 & 9 & 8.3 & $3.8-15.1$ & 113 & 18 & 15.9 & $9.7-24.0$ \\
\hline Morogoro & 1556 & 187 & 12.0 & $10.4-13.7$ & 1630 & 147 & 9.0 & $7.7-10.5$ \\
\hline aHembeti & 81 & 9 & II.I & $5.2-20.0$ & 99 & 2 & 2.1 & $0.2-7.1$ \\
\hline aMkuyuni & 154 & 5 & 3.3 & I.I - 7.4 & 173 & 3 & 1.7 & $0.4-5.0$ \\
\hline cMorogoro & 550 & 54 & 9.8 & $7.5-12.6$ & 559 & 64 & 11.5 & $8.9-14.4$ \\
\hline cUhuru & 771 & 119 & 15.4 & $13.0-18.2$ & 799 & 78 & 9.8 & $7.8-12.0$ \\
\hline Tanga & 1986 & 65 & 3.3 & $2.5-4.2$ & 2084 & 191 & 9.2 & $8.0-10.5$ \\
\hline bHandeni & 380 & 8 & 2.1 & $0.9-4.1$ & 403 & 32 & 7.9 & $5.5-11.0$ \\
\hline aKwamkono & 158 & 16 & 10.1 & $5.9-15.9$ & 168 & 8 & 4.7 & $2.1-9.2$ \\
\hline bLushoto & 237 & I & 0.4 & $0.0-2.3$ & 246 & 13 & 5.3 & $2.8-8.9$ \\
\hline aMagoma & 171 & 7 & 4.1 & $1.7-8.3$ & 175 & 2 & I.I & $0.1-4.1$ \\
\hline cMakorola & 518 & 8 & 1.5 & $0.7-3.0$ & 545 & 36 & 6.6 & $4.7-9.0$ \\
\hline cNgamiani & 522 & 25 & 4.8 & $3.1-69.9$ & 547 & 100 & 18.3 & $15.1-21.8$ \\
\hline
\end{tabular}

Key: arural, bsemi-urban, curban, dborder, eroadside

previous years, in contrast to women with HIV, women with no education were more likely to be infected with syphilis than were women with some education ( $\mathrm{p}<$ 0.0001 ) (Fig 5). Overall, 130/17,813 (0.7\%) of clinic attendees were co-infected with syphilis and HIV.

\section{Discussion}

The prevalence of HIV infection in this surveillance round was $8.7 \%$, which compares with the findings of a population survey showing the prevalence of HIV to be $7.3 \%$ among adult Tanzanians aged 15-49 years [7]. The peak age was 25 to 34 years, which may explain the highest HIV prevalence among women with one or two previous pregnancies compared to those with more (Fig 6).

HIV infection show strong regional variations, with Mbeya being the most affected (15.7\%) and Dar es

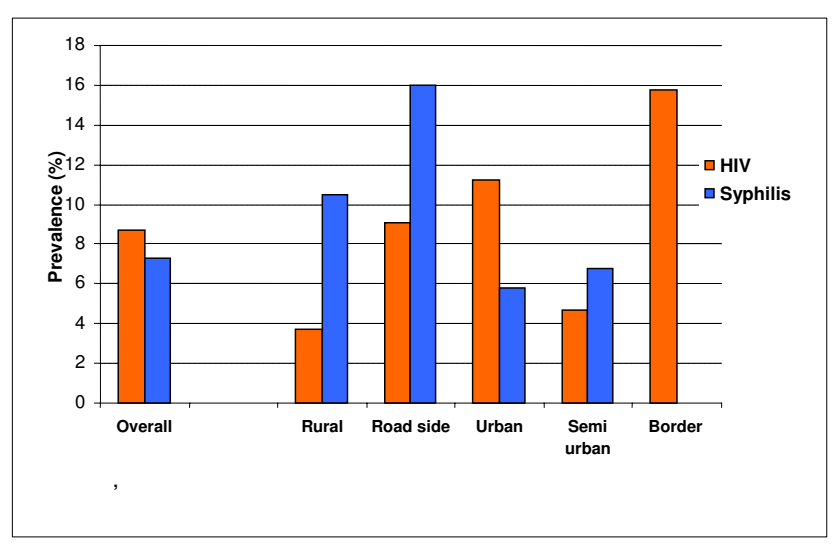

Figure 2

Prevalence of HIV and syphilis among ANC attendees by ANC location.
Salaam $(10.8 \%)$, the capital city, being second most affected region. These data are in keeping with the previous ANC surveillance data (Table 2) and recent reports of the general population survey, showing that these regions have the highest HIV prevalence in Tanzania[7]. Remarkably, Kagera, a region where the HIV/AIDS epidemic started in Tanzania and where the prevalence of HIV was high for sometime[8] had the lowest prevalence of HIV infection, an observation also been seen in population surveys [7].

Significantly, the HIV prevalence of women attending road side, border and urban ANC clinics was higher than rural attendees, an observation that is consistent with past [8-11] and recent studies [7]. These differences have been linked with differences in community factors such as the level of trade, social and economic activity [12], ratio of

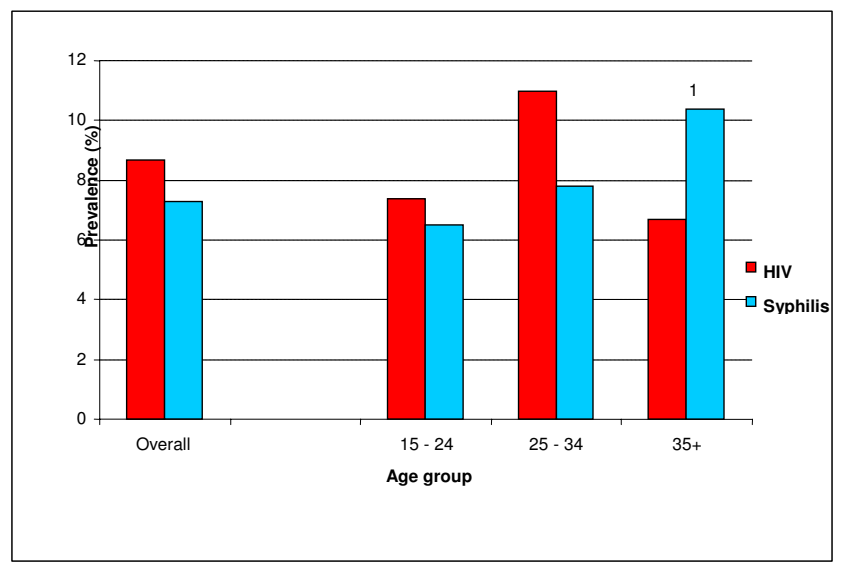

Figure 3

Prevalence of HIV and syphilis by age group among ANC attendees, Tanzania 


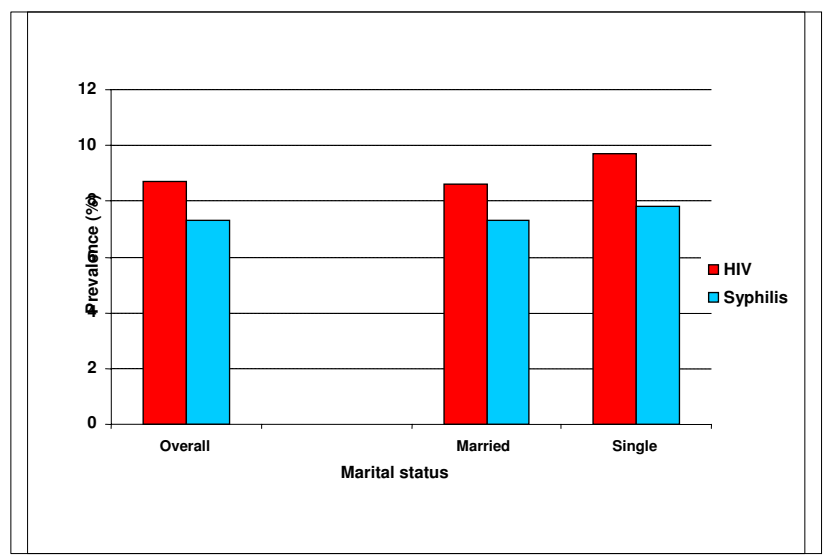

\section{Figure 4}

Prevalence of HIV and syphilis among ANC attendees by marital status.

bar workers per male population, level of community mobility, and distance to the nearest town [16]. There was slightly higher prevalence of HIV among single than married women, which was statistically not significant, which is in keeping with the previous ANC surveillance report (Table 2). Other studies in Tanzania have found highest HIV prevalence among married women or formerly married women $[7,9]$. Such variations may imply that marital status per se is not an indicator of sexual activity and hence risk for HIV infection, which indicate the need to consider risk of their partners as well [14].

This surveillance showed that HIV prevalence increased with the level of education, from 5.2\% among women with no education to $9.3 \%$ with women with primary or

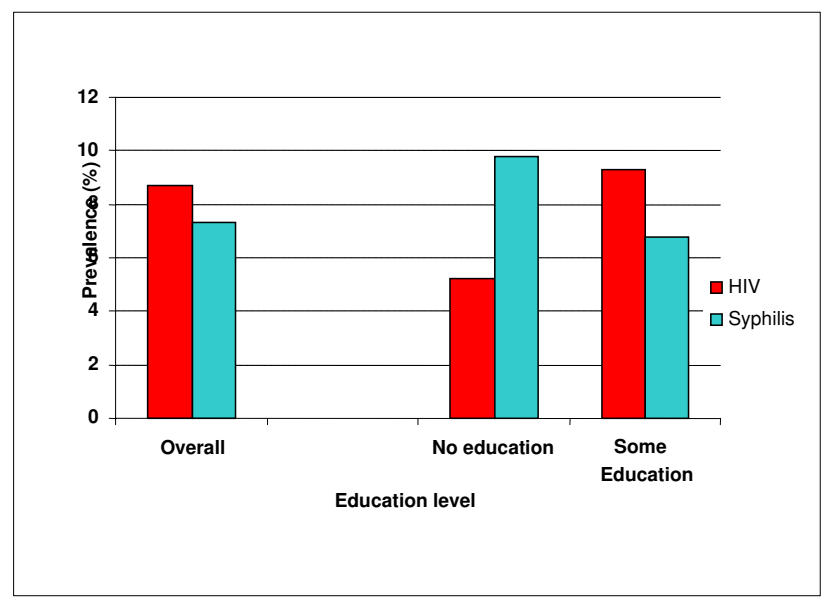

\section{Figure 5}

Prevalence of HIV and syphilis among ANC Attendees by education status, Tanzania, 2003/04.

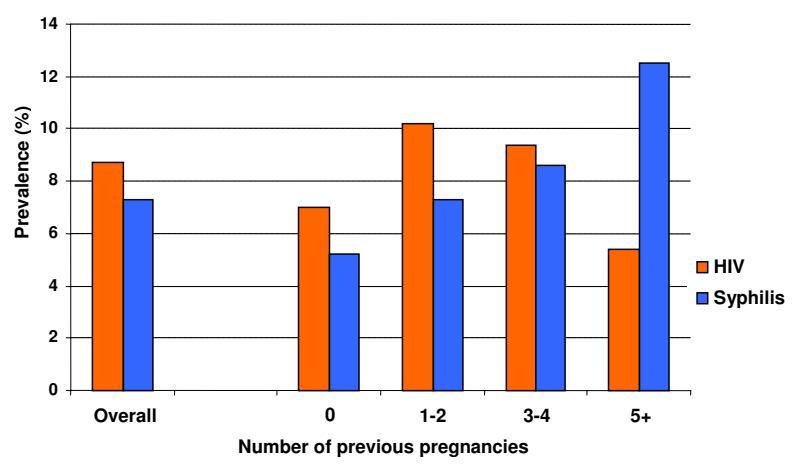

\section{Figure 6}

Prevalence of HIV and syphilis among ANC attendees by number of previous pregnancies, Tanzania, 2003/04.

higher level of education, which was also observed in the previous ANC surveillance (Table 2) and other studies $[7,14]$. This could be due to the fact the educated, through their access to income and their position in society, engage in sexual behaviours which place themselves and their spouses at risk of HIV infection. The high level of HIV prevalence among the educated individuals will lead to substantial economic losses through the erosion of Africa's most able and most educated segment of the population.

When the HIV results of this surveillance are compared with those of the first ANC survey conducted in 2001/ 2002 (Table 2) we notice a decrease in prevalence in Dar es Salaam and Mtwara, with the other regions have no change. The HIV prevalence among women attending border clinics fell from $17.3 \%$ to $15.3 \%$. These changes were rather small and are probably a result of random variation in the different regions and sites. As with the previous ANC surveillance, the age of peak HIV prevalence was 25-34 years compared with 30-34 for females in the population survey.

The overall prevalence of syphilis was $7.3 \%$, which compares with $8.2 \%$ reported in the last surveillance conducted in 2001/2002 (Table 2). Like HIV, the syphilis data show strong geographical variations, ranging from $2.1 \%$ in Kigoma to $14.9 \%$ in Kagera. There were also notable observations from the syphilis data including higher syphilis prevalence in women from roadside clinics. Unlike HIV, syphilis was higher among women without formal education and those aged $\geq 34$ years, and there was no association with marital status. These observations are in keeping with previous ANC surveillance reports (Table 
Table 2: HIV data of the ANC surveillance studies conducted in 200I/2002 and 2003/2004

\begin{tabular}{|c|c|c|}
\hline & ANC Surveillance $2003 / 2004$ & ANC Surveillance $2001 / 2002$ \\
\hline \multicolumn{3}{|l|}{ Region } \\
\hline Dar es Salaam & $10.8 \%$ & $12.8 \%$ \\
\hline Dodoma & $7.8 \%$ & $6.1 \%$ \\
\hline Kagera & $4.7 \%$ & $5.6 \%$ \\
\hline Kilimanjaro & $5.7 \%$ & $6.3 \%$ \\
\hline Mbeya & $15.7 \%$ & $16 \%$ \\
\hline Mtwara & $5.1 \%$ & $7.1 \%$ \\
\hline Kigoma & $5.1 \%$ & ND \\
\hline Lindi & $7.1 \%$ & ND \\
\hline Morogoro & $9.0 \%$ & ND \\
\hline Tanga & $9.2 \%$ & ND \\
\hline \multicolumn{3}{|l|}{ Residence } \\
\hline Urban & $11.2 \%$ & $12.1 \%$ \\
\hline Semi-urban & $4.7 \%$ & $3.7 \%$ \\
\hline Roadside & $9.1 \%$ & $10.3 \%$ \\
\hline Border & $15.3 \%$ & $17.3 \%$ \\
\hline Rural & $3.7 \%$ & $4.1 \%$ \\
\hline Peak age & $25-34$ & $25-34$ \\
\hline \multicolumn{3}{|l|}{ Education } \\
\hline None & $5.2 \%$ & $6.4 \%$ \\
\hline Some education & $9.3 \%$ & $10.2 \%$ \\
\hline \multicolumn{3}{|l|}{ Marital status } \\
\hline Married & $8.6 \%$ & $9.1 \%$ \\
\hline Single & $9.7 \%$ & $12.6 \%$ \\
\hline Overall Prevalence & $8.7 \%$ & $9.6 \%$ \\
\hline
\end{tabular}

$N D=$ No Data

3). Collectively these findings indicate that syphilis is associated with poverty, inadequate access to health care, and lack of education

Remarkably, some regions with low HIV prevalence e.g. Kagera had very high prevalence of syphilis, and vice-versa for Mbeya, Dar es Salaam and Tanga regions and there were also very significant variations even in areas that were geographically close to each other, such as the studied areas in Dar es Salaam, Dodoma and Kagera, without corresponding differences in HIV prevalence (Table 1). The syphilis data need to be interpreted with caution for several reasons including; 1) the use of RPR without confirmatory testing with a more specific test for syphilis such as FTA-ABS or TPHA, testing [15] or antitreponemal IgM ELISAs [16] 2) lack of quality control measures 3) although RPR test is easy to perform and inexpensive it may be difficult to interpret and requires training of health personnel (West et al), which may have been especially the case in the rural areas where testing was done by inexperienced ANC nurses, 4) false negatives may occur both in early primary cases and in patients with secondary syphilis as a result of prozone reactions (Berkowitz 1990), and 5) poor performance of RPR test under field condi- tions that are typical of many developing country health centres [17-19] due to temperature, dust and the light conditions $[17,20,21]$. Future surveillance for syphilis infection should address these issues in order to improve the quality of the syphilis data.

\section{Conclusion}

The high HIV prevalence observed among the ANC clinic attendees in Tanzania call for increase in voluntary counselling and testing (VCT) services and access to antiretroviral drugs (ARV) in the clinics. There is also a need for modification of obstetric practices and infant feeding options in HIV infection in order to prevent mother to child transmission of HIV. To increase uptake to HIV testing the opt-out strategy in which all clients are offered HIV testing is recommended. Post-test counselling of HIV-negative women should also be carried out to prevent new HIV infections. To this end we are suggesting; i) scaling up existing prevention of mother-to-child transmission (PMTCT) programs by rapidly mobilizing resources, ii) providing technical assistance and expanded training for health care providers (including family planning providers, traditional birth attendants, and others) on appropriate antenatal care, safe labor and delivery practices and 
Table 3: Syphilis data of the ANC surveillance studies conducted in 2001/2002 and 2003/2004

\begin{tabular}{|c|c|c|}
\hline & ANC Surveillance $2003 / 2004$ & ANC Surveillance $2001 / 2002$ \\
\hline \multicolumn{3}{|l|}{ Region } \\
\hline Dar es Salaam & $5.1 \%$ & $4.8 \%$ \\
\hline Dodoma & $8.4 \%$ & $6.1 \%$ \\
\hline Kagera & $14.9 \%$ & $9.3 \%$ \\
\hline Kilimanjaro & $6.3 \%$ & $3.0 \%$ \\
\hline Mbeya & $6.2 \%$ & $10.7 \%$ \\
\hline Mtwara & $9.1 \%$ & $10.5 \%$ \\
\hline Kigoma & $2.1 \%$ & ND \\
\hline Lindi & $7.1 \%$ & ND \\
\hline Morogoro & $12.0 \%$ & ND \\
\hline Tanga & $3.3 \%$ & ND \\
\hline \multicolumn{3}{|l|}{ Residence } \\
\hline Urban & $5.8 \%$ & $5.1 \%$ \\
\hline Semi-urban & $6.8 \%$ & $13.3 \%$ \\
\hline Roadside & $16.0 \%$ & $9.7 \%$ \\
\hline Border & $7.0 \%$ & $13.4 \%$ \\
\hline Rural & $10.5 \%$ & $4.1 \%$ \\
\hline Peak age & +35 & +35 \\
\hline \multicolumn{3}{|l|}{ Education } \\
\hline None & $9.8 \%$ & $11.5 \%$ \\
\hline Some education & $6.8 \%$ & $7.5 \%$ \\
\hline \multicolumn{3}{|l|}{ Marital status } \\
\hline Married & $7.3 \%$ & $8.2 \%$ \\
\hline Single & $7.8 \%$ & $8.2 \%$ \\
\hline Overall Prevalence & $7.3 \%$ & $7.2 \%$ \\
\hline
\end{tabular}

ND $=$ No Data

breast-feeding, iii) strengthening the referral links among health care facilities and providers, iv) ensuring effective supply chain management of the range of PMTCT-related products and equipment, and v) expanding PMTCT programs to include ARV treatment for eligible HIV-infected mothers and other members of the child's immediate family (commonly known as "PMTCT-plus").

\section{Competing interests}

The author(s) declare that they have no competing interests.

\section{Authors' contributions}

Authors contributed equally to this work. All authors read and approved the final manuscript.

\section{Acknowledgements}

We wish to acknowledge with sincere gratitude to all those who contributed in this survey. Our thanks go to all health care providers at the regional, district and health care facility levels who have given so earnestly of their time and energy. Their contributions made this report possible. We acknowledge with special gratitude the financial contributions provided by the international partners. These include the US Centres for Disease Control and Prevention (CDC), Joint United Nations Programme on AIDS (UNAIDS), World Health Organisation (WHO) and United Nations Devel- opment Project (UNDP). We specifically wish to thank the CDC for their technical collaboration.

\section{References}

I. [http://www.tanzania.go.tz/hiv aids.html].

2. United Republic of Tanzania. Ministry of Health. National AIDS Control Programme. Surveillance of HIV and syphilis among antenatal clinic enrolees 200I-2002.

3. United Republic of Tanzania. Demographic and Health Survey 19961997.

4. United Republic of Tanzania. Ministry of Health. National STD Treatment Guidelines 2002.

5. Dean AG: Epi Info ${ }^{\mathrm{TM}}$ and Epi Map: Current status and plans for Epi Info ${ }^{\mathrm{TM}}$ 2000. J Pub Health Management and Practice 1999, 5:54-57.

6. [http://www.stata.com].

7. Tanzania Commision for AIDS, National Bureau of Statistics and ORC Macro. HIVIAIDS Indicator Survey 2003-2004 2005.

8. Killewo J, Nyamuryekunge K, Sandstrom A: Prevalence of HIV-I infection in the Kagera region of Tanzania: a populationbased study. AIDS 1990, 4:108I-1085.

9. Mnyika KS, Klepp KI, Kvale G, Nilssen S, Kissila PE, Ole-King'ori N Prevalence of HIV-I infection in urban, semi-urban and rural areas in Arusha region, Tanzania. AIDS 1994, 8:1477-1481.

10. Killewo J, Dahlgren L, Sandstrom A: Socio-geographical patterns of HIV-I transmission in Kagera Region, Tanzania. Soc Sci Med 1994, 38: 129-134.

II. Barongo LR, Borgdorff MW, Mosha FF: The epidemiology of HIV$I$ infection in urban areas, roadside settlements and rural villages in Mwanza Region, Tanzania. AIDS 1992, 6:1521-1528. 
12. Bloom SS, Urassa M, Isingo R, Ng'weshemi J, Boerma JT: Community effects on the risk of HIV infection in rural Tanzania. Sex Transm Infect 2002, 78:26I-266.

13. Mwaluko G, Urassa M, Isingo R, Zaba B, Boerma JT: Trends in HIV and sexual behaviour in a longitudinal study in a rural population in Tanzania, 1994-2000. AIDS 2003, 17:2645-265I.

14. Kapiga SH, Shao JF, Lwihula GK, Hunter DJ: Risk factors for HIV infection among women in Dar-es-Salaam, Tanzania. J Acquir Immune Defic Syndr 1994, 7:30I-9.

15. Larsen S, Steiner B, Rudolph A: Laboratory diagnosis and interpretation of tests for syphilis. Clin Microbiol Rev 1995, 8: I-2I.

16. Young H: Syphilis: new diagnostic directions. Int J STD AIDS 1992, 3:913-7.

17. West B, Walraven G, Morison L, Brouwers J, Bailey R: Performance of the rapid plasma reagin and the rapid syphilis screening tests in the diagnosis of syphilis in field conditions in rural Africa. Sex Transm Inf 2002, 78:282-5.

18. Berkowitz K, Baxi L, Fox HE: False negative syphilis screening: the prozone phenomenon, non-immune hydrops, and diagnosis of syphilis during pregnancy. Am J Obstet Gynecol 1990 163:975-7.

19. Van Dyck E, Van de Velden L, Ndoye I: Evaluation of the RPR "teardrop" card test for screening for syphilis in field conditions. Sex Transm Dis 1993, 20:194-7.

20. Creighton ET: Rapid plasma reagin (RPR) I8-mm circle card test. In A manual of tests for syphilis 8th edition. Edited by: Larsen SA, Hunter EF, Kraus SJ. Washington, DC: American Public Health Association; 1990:100-8.

21. El Zaatari M, Martens M: False negative syphilis screening due to change in temperature. Sex Transm Dis 1994, 21:243-6.

\section{Pre-publication history}

The pre-publication history for this paper can be accessed here:

http://www.biomedcentral.com/1471-2458/6/91/prepub

Publish with Biomed Central and every scientist can read your work free of charge

"BioMed Central will be the most significant development for disseminating the results of biomedical research in our lifetime. "

Sir Paul Nurse, Cancer Research UK

Your research papers will be:

- available free of charge to the entire biomedical community

- peer reviewed and published immediately upon acceptance

- cited in PubMed and archived on PubMed Central

- yours - you keep the copyright 